\title{
A comparison of two functional tests in quadriplegia: The quadriplegia index of function and the functional independence measure
}

\author{
N Yavuz ${ }^{1}$, M Tezyürek ${ }^{2}$ and M Akyüz ${ }^{2}$ \\ ${ }^{1}$ Department of Physical Medicine and Rehabilitation, Baskent University School of Medicine, Geriatric and \\ Psycho-social Rehabilitation Center, Ankara and ${ }^{2}$ Ankara Rehabilitation Center, Ankara, Turkey
}

\begin{abstract}
Individuals with spinal cord injury are evaluated according to a set of guidelines based on motor, sensory, and functional tests. The resulting scores are used to quantify the extent of neurological injury and functional loss. The purpose of the present study was to compare certain scoring systems using the same group of patients. Twenty-nine subjects with cervical spine cord injury were evaluated by the same examiner using three scales: (1) The American Spinal Cord Injury Association (ASIA) (2) The Quadriplegia Index of Function (QIF) (3) The Functional Independence Measure (FIM) Assessments were made both at admission to, and discharge from, the rehabilitation center. Positive change in motor score is widely used as an indicator of recovery after spinal cord injury. We assessed the relationship of the two functional tests, the FIM and the QIF, to ASIA scores and found strong correlations in both cases. The feeding and dressing categories of QIF showed an even stronger correlation to ASIA motor scores, though the statistical significance was the same for corresponding categories of FIM and QIF. The percent of recovery on ASIA motor scores was significantly correlated only to gain in QIF scores, not FIM. FIM lacks the category of bed activities. Some additions to the FIM may be useful, especially in the feeding and dressing categories, and a category of bed activities could be included as well, in order to improve sensitivity.
\end{abstract}

Keywords: spinal cord injury; quadriplegia; functional assessment; evaluation

\section{Introduction}

Spinal cord injury is a traumatic event which results in motor, sensory and autonomic dysfunction. Typically, the patient can only achieve a fairly low degree of success in daily living tasks and faces severe adaptation problems. Traumatic spinal cord injury occurs primarily in young adults, and the impact of this catastrophic injury affects every aspect of their lives. In recent decades, paralleling the improvements in medicine and technology, the mortality rate due to spinal cord injury has been decreasing. The survival rate has been on the rise especially among those with cervical cord lesions, so the concept of the quadriplegic patient's quality of life has become the key issue. The aim of rehabilitation today should be to maximize patient performance in self-care and daily living activities.

Accurate documentation of the functional capacity of a tetraplegic patient is essential in order to predict outcome, measure the effect of therapeutic interventions, and provide the necessary information to both patient and family.

Among the currently used tools for evaluating a quadriplegic patient's functional performance are the

Correspondence: $\mathrm{N}$ Yavuz, Baskent Universitesi Tip Fakultesi, 1 Cadde No: 77 Kat: 4, Bahcelievler, 06490, Ankara, Turkey modified Barthel index, the PULSES profile, the Kenny self-care evaluation, the Functional Independence Measure (FIM), and the Quadriplegia Index of Function (QIF). Few studies in the literature compare the sensitivity of these instruments ${ }^{1-3}$ and their correlation to motor performance. ${ }^{4-6}$ Positive change in motor score is widely used as an indicator of recovery after spinal cord injury. ${ }^{4-6}$ Marino et $a l^{4}$ related strength to function by correlating upper extremity motor scores to the feeding, grooming, and bathing categories of FIM and the corresponding categories of QIF in a group of patients.

In this study, we evaluated 29 tetraplegic patients according to ASIA, FIM and QIF. We assessed the relationship of the two functional tests, FIM and QIF, to ASIA scores and investigated which functional test was more strongly correlated to ASIA motor scores. We also correlated the percentage improvement in overall ASIA motor scores to FIM and QIF scores. Our study revealed that ASIA motor scores were strongly correlated to total FIM and QIF scores, and that the correlation coefficients were almost identical for QIF and FIM. When we considered percentage improvement, we realized that the per cent gain on ASIA motor and QIF were strongly correlated, whereas this was not the case for per cent gain on ASIA motor and FIM. Other evidence also pointed to 
QIF's superiority over FIM; the positive influence of early rehabilitation on motor recovery was reflected in functional performance by QIF but not by FIM.

\section{Methods}

Twenty male and nine female, consecutively seen, patients who had become tetraplegic due to traumatic spinal cord injury were included in this study. All were admitted to the Ankara Rehabilitation Center between May 1994 and January 1996. Their ages ranged from 14-66 years (mean, 37 years) and length of hospital stay averaged 18 weeks (range, $2-37$ weeks). The time from onset of injury to admission ranged from 2-72 weeks (mean, 20 weeks) and the leading causes of injury were auto accidents $(51.7 \%)$ and falls $(13.8 \%)$. At the rehabilitation center, all patients were evaluated by the same physiatrist at admission, and all but one were seen by this same individual at discharge. The battery of tests consisted of ASIA motor, light touch and pinprick sensation, the FIM, and the QIF. The distribution of patients according to neurologic level of injury is shown in Table 1. According to the ASIA 1992 system, 18 patients had complete, and 11 had incomplete spinal cord injuries. ${ }^{7}$

The ASIA motor index score uses standard manual muscle testing on a six-grade scale from zero to five. The key upper extremity muscles representing the five consecutive segments between $\mathrm{C} 5$ and $\mathrm{T} 1$ are elbow flexors, wrist extensors, elbow extensors, finger flexors, and finger adductors. The maximum total upper extremity motor score (UEMS) is 50 points bilaterally. The key lower extremity muscles representing the five consecutive segments between L2 and S1 are hip flexors, knee extensors, ankle dorsiflexors, long toe extensors, and ankle plantar flexors. Total lower extremity motor score (LEMS) is 50 points bilaterally, making the overall ASIA motor score 100 points. Sensory scores are tested for 28 dermatomes on a three-point scale (0: absent, 1: impaired, 2: normal), making a total score of 112 bilaterally for both light touch and pinprick sensation. ${ }^{7}$

The FIM consists of 18 items organized under six categories of function. Each item is scored from one to seven. The categories include self-care activities (eating, grooming, bathing, upper and lower body dressing, and toileting); sphicter control (bowel and

Table 1 Patient distribution according to neurological level

\begin{tabular}{lcr}
\hline Neurological level & Number of patients & $\%$ \\
\hline C3 & 1 & 3.44 \\
C4 & 1 & 3.44 \\
C5 & 10 & 34.48 \\
C6 & 11 & 37.96 \\
C7 & 2 & 6.90 \\
C8 & 3 & 10.34 \\
T1 & 1 & 3.44 \\
\hline
\end{tabular}

bladder management); mobility (transfers to toilet, bed, chair, tub); locomotion (walking, wheelchair, stairs); communication (comprehension, expression); and social cognition. The maximum score is 126 points. ${ }^{8}$ FIM reflects an appreciation of the multidimentional nature of factors.

The QIF was developed to document small but significant functional gains made by tetraplegics during medical rehabilitation. ${ }^{1}$ It takes 30 minutes to perform, but this is still quite long compared to the FIM. The QIF is comprised of 10 variables: transfers, grooming, bathing, dressing, feeding, mobility, bed activities, bladder program, bowel program, and understanding of personal care. The last category consists of a questionnaire which assesses the patient's understanding of skin care, nutrition, equipment, medication, infections etc. The first nine categories assess the patient's functional performance. Each functional category addresses several specific tasks, and each task is scored from zero to four in order of increasing independence. If a specific task cannot be tested on the patient, nine points are scored for that item (ie., for an ambulatory patient, the item of wheelchair mobility is not applicable).

For the categories of bladder and bowel program, the item appropriate for the patient is chosen from the list of options, and only this item is scored. The functional score of each category is calculated according to the weighted sums of specific items (Table 2). The total score from nine functional categories is 180 points, and the score of understanding of personal care is 20 points. This total of 200 is then divided by two to determine the score out of 100 points. This study used the weighted scores for each QIF category, the total being out of 180 points.

For statistical analysis we used Spearman's correlation coefficients. We correlated UEMS to the grooming, bathing, and feeding categories of both the FIM and QIF. We correlated total motor scores with the categories of dressing, transfer, locomotion,

Table 2 Weighted scores for each category in the QIF

\begin{tabular}{lcc}
\hline Category & Number of items & Weighted sum \\
\hline Transfers & 8 & 16 \\
Grooming & 3 & 12 \\
Bathing & 4 & 8 \\
Feeding & 7 & 24 \\
Dressing & 6 & 20 \\
Wheelchair mobility & 7 & 28 \\
Bed activities & 5 & 20 \\
Bladder program & Chosen from 6 sets & 28 \\
Bowel program & Chosen from 4 sets & 24 \\
Functional Total & & 180 \\
Understanding & & \\
$\quad$ personal care & 10 & 20 \\
QIF Total & &
\end{tabular}


and bladder and bowel program for both functional tests. We also correlated the FIM and QIF scores of corresponding categories. Since we had applied the battery of tests both at admission and discharge, we were able to correlate percentage of motor recovery with percentage improvement on FIM and QIF scores. The Mann-Whitney U-test was used to assess statistical significance.

\section{Results}

All 28 patients who were evaluated at admission and discharge made considerable functional gains according to FIM and QIF, and some improvement was also noted on the ASIA motor and sensory scales. The average gain between the two evaluations was 9.83 points for motor score, 12.43 points for light touch sensation, and 9.14 points for the pinprick sensation. When we assessed functional gains made by the patients, the average gain was 30.12 points on QIF and 14.20 points on FIM score.

The distribution of average scores among the complete and incomplete tetraplegics are shown in Table 3. The improvement shown by incomplete tetraplegic patients on the ASIA motor, QIF, and FIM scores was significant $(P<0.01)$ when compared to the complete tetraplegic patients (Mann-Whitney U-test).

The total scores on the two functional assessment instruments, QIF and FIM, correlated strongly $(r=0.97, \quad P<0.001) \quad$ with each other and also displayed good correlation to ASIA motor, pinprick, and light touch sensation scores (Table 4).

In order to compare the different categories of the two functional tests according to their relationship to motor performance, the corresponding categories were chosen and included in the statistical analyses (Spearman's correlation coefficient). In light of the fact that self-care tasks such as grooming, bathing and feeding require primarily upper extremity function, we correlated the scores of the abovementioned categories to UEMS. Table 5 shows these correlations alongside those reported by Marino et $a l .{ }^{4}$

For the other categories, such as dressing, transfers, locomotion (mobility), and bladder and bowel management, we correlated each score to the wholebody ASIA motor score (Table 6). The correlation coefficients for the FIM and QIF categories were almost identical.

The average length of stay in the rehabilitation center was $18+10.29$ weeks. When we grouped the patients as having stayed in the hospital for more versus less than 18 weeks, we failed to find any statistical significance between the two groups in terms of improvement on QIF and FIM scores. The recovery in motor scores was also insignificant $(P>0.05$, MannWhitney U-test). When we grouped the patients according to age (younger and older than 40 years), there was no significant difference between the groups indicated by either FIM or QIF $(P>0.05$, MannWhitney U-test). Early rehabilitation had a significant influence on ASIA motor recovery, and this was reflected by QIF $(P<0.005)$ but not by FIM $(P>0.05$, Mann-Whitney U-test) when patients were grouped as having had an interval of more versus less than 3 months before admission and after the onset of tetraplegia.

We also calculated the per cent improvement for each patient in terms of their total QIF, FIM, and ASIA motor scores. Surprisingly, the per cent improvement indicated by the ASIA motor score correlated strongly with the per cent gain in QIF $(r=0.68, \quad P=0.001)$ but did not exhibit such a significant correlation with gain in the FIM score $(r=0.38, P>0.05)$.

\section{Discussion}

The aim of this study was to determine the relationship between functional tests and motor performance in quadriplegic patients, and to investigate which of the

Table 4 Correlation of ASIA scores with FIM and QIF scores (Spearman)

\begin{tabular}{lccccc}
\hline ASIA score & \multicolumn{2}{c}{ QIF functional score } & \multicolumn{2}{c}{ FIM score } \\
\hline ASIA motor & $r: 0.91$ & $P<0.001$ & $r: 0.91$ & $P<0.001$ \\
ASIA light touch & $r: 0.64$ & $P<0.001$ & $r: 0.58$ & $P<0.01$ \\
ASIA pinprick & $r: 0.65$ & $P<0.01$ & $r: 0.55$ & $P<0.01$ \\
\hline
\end{tabular}

Table 3 Improvement of complete and incomplete quadriplegics according to the ASIA system, the FIM, and the QIF

\begin{tabular}{|c|c|c|c|c|}
\hline & Test & $\begin{array}{c}\text { Average score at } \\
\text { admission }\end{array}$ & $\begin{array}{c}\text { Average score at } \\
\text { discharge }\end{array}$ & $\begin{array}{c}\text { Average improvement } \\
\text { in score }\end{array}$ \\
\hline & ASIA motor & $21.1 \pm 7.3$ & $24.8 \pm 8.8$ & 3.6 \\
\hline Complete & ASIA light touch & $30.5 \pm 13.5$ & $37.5 \pm 22.6$ & 7 \\
\hline \multirow[t]{3}{*}{ Quadriplegics } & FIM & $52.7 \pm 10.5$ & $59.1 \pm 12.7$ & 6.4 \\
\hline & QIF & $25.7 \pm 28.7$ & $39.0 \pm 31.2$ & 13.3 \\
\hline & ASIA motor & $68.54 \pm 16.3$ & $81.58 \pm 11.8$ & 13.04 \\
\hline Incomplete & ASIA light touch & $77.3 \pm 20.9$ & $93.3 \pm 21.6$ & 16.0 \\
\hline \multirow[t]{2}{*}{ Quadriplegics } & FIM & $79.5 \pm 24.5$ & $98.4 \pm 23.3$ & 18.9 \\
\hline & QIF & $111.3 \pm 68.5$ & $151.4 \pm 67.7$ & 40.1 \\
\hline
\end{tabular}


two functional assessments, QIF or FIM, better reflects the small functional gains made by patients.

ASIA is a good system for evaluating spinal cord injury patients. Allison indicated that correlation of level of injury to functional performance was not the appropriate approach, since a patient may be complete or incomplete at the same neurologic level. ASIA has been labeled the best method to evaluate recovery in spinal cord-injured patients. ${ }^{9-13}$ Many studies in the literature have shown a positive correlation between function and strength., 4,14 Our investigation also indicated that both QIF and FIM are highly correlated to ASIA motor scores.

Marino et $a l^{4}$ evaluated 22 C4-C7 Frankel A-D, medically stable spinal cord-injured patients using only self-care categories, such as feeding, grooming, and bathing categories of FIM and QIF (Partial FIM and QIF). They related the scores of these categories to $\mathrm{UEMS}^{4}$ and found a good correlation between UEMS and both partial FIM and QIF scores. In their study, the feeding category of QIF was strongly correlated with UEMS, whereas this was not the case for FIM. They reported as well that in the bathing and grooming categories, FIM was also strongly correlated with motor scores (Table 5). Similarly, in our study QIF feeding scores were more strongly correlated (0.84) with UEMS, but the difference between the correlation coefficients was not striking and $P$ was $<0.001$ for both. Likewise, in the study by Marino et $a l^{4}$ there was no statistical significance between scores on the feeding categories of both tests $(P<0.01$ for both). These reseachers held that QIF assessed functional ability in feeding more accurately than did FIM, and attributed the success of QIF to its questioning more specific tasks in each category.

Our study correlated the other corresponding categories of FIM and QIF with total ASIA motor scores. There were strong correlations in the categories of dressing, transfer, locomotion, and bladder and bowel management. In fact, the correlation coefficients were almost identical in most cases. In the dressing category the correlation coefficients were 0.91 for QIF and 0.80 for FIM, indicating a stronger correlation between ASIA and QIF. As in the feeding category, the difference was not statistically significant, with $P<0.001$ for both (Table 6).

In the feeding and dressing categories, gains on QIF correlated more strongly than FIM to gains in motor scores. The distribution of our patients on the basis of FIM and QIF scores in the feeding and dressing categories are shown in Figures 1-4. A more homogeneous distribution in the QIF scores is noted, and this may be attributed to the evaluation of more specific tasks using QIF. ${ }^{4}$ With QIF the feeding

Table 5 Spearman correlation of QIF, FIM, and ASIA upper extremity motor scores (UEMS) in self-care categories

\begin{tabular}{|c|c|c|c|c|c|c|}
\hline Category & $U E M S$ vs $Q I F$ & $\begin{array}{l}\text { Present study } \\
\text { UEMS vs FIM }\end{array}$ & $Q I F$ vs $F I M$ & $U E M S$ vs $Q I F$ & $\begin{array}{l}\text { Marino et } \mathrm{al}^{4} \\
U E M S \text { vs } F I M\end{array}$ & $Q I F$ vs $F I M$ \\
\hline Grooming & $\begin{aligned} r & =0.85 \\
P & <0.001\end{aligned}$ & $\begin{aligned} r & =0.83 \\
P & <0.001\end{aligned}$ & $\begin{aligned} r & =0.91 \\
P & <0.001\end{aligned}$ & $r=0.90$ & $r=0.91$ & $r=0.94$ \\
\hline Bathing & $\begin{aligned} r & =0.75 \\
P & <0.001\end{aligned}$ & $\begin{aligned} r & =0.76 \\
P & <0.001\end{aligned}$ & $\begin{aligned} r & =0.96 \\
P & <0.001\end{aligned}$ & $r=0.84$ & $r=0.75$ & $r=0.92$ \\
\hline Feeding & $\begin{aligned} r & =0.84 \\
P & <0.001\end{aligned}$ & $\begin{aligned} r & =0.76 \\
P & <0.001\end{aligned}$ & $\begin{aligned} r & =0.91 \\
P & <0.001\end{aligned}$ & $r=0.90^{*}$ & $r=0.53^{*}$ & $r=0.75$ \\
\hline
\end{tabular}

$* P<0.01$

Table 6 Spearman correlations of QIF, FIM, and ASIA motor scores in other categories

\begin{tabular}{lccc}
\hline Category & $A S I A$ vs $Q I F$ & $A S I A$ vs $F I M$ & $Q I F$ vs $F I M$ \\
\hline Dressing & $r=0.91$ & $r=0.80$ & $r=0.88$ \\
& $P<0.001$ & $P<0.001$ & $P<0.001$ \\
Transfers & $r=0.82$ & $r=0.80$ & $r=0.99$ \\
& $P<0.001$ & $P<0.001$ & $P<0.001$ \\
Mobility & $r=0.90$ & $r=0.86$ & $r=0.96$ \\
Bladder & $P<0.001$ & $P<0.001$ & $P<0.001$ \\
Program & $r=0.79$ & $r=0.77$ & $r=0.87$ \\
Bowel & $P<0.001$ & $P<0.001$ & $P<0.001$ \\
Program & $r=0.79$ & $r=0.74$ & $r=0.88$ \\
& $P<0.001$ & $P<0.001$ & $P<0.001$ \\
\hline
\end{tabular}

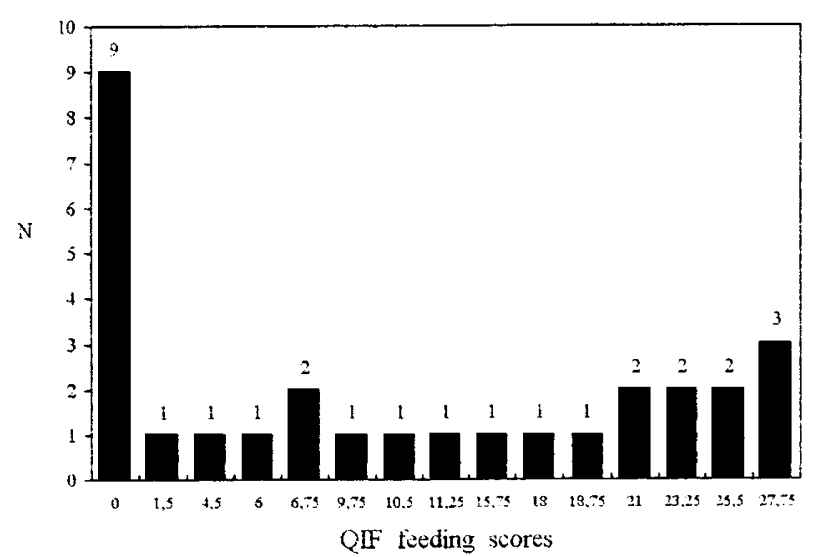

Figure 1 Distribution of patients according to QIF feeding score 


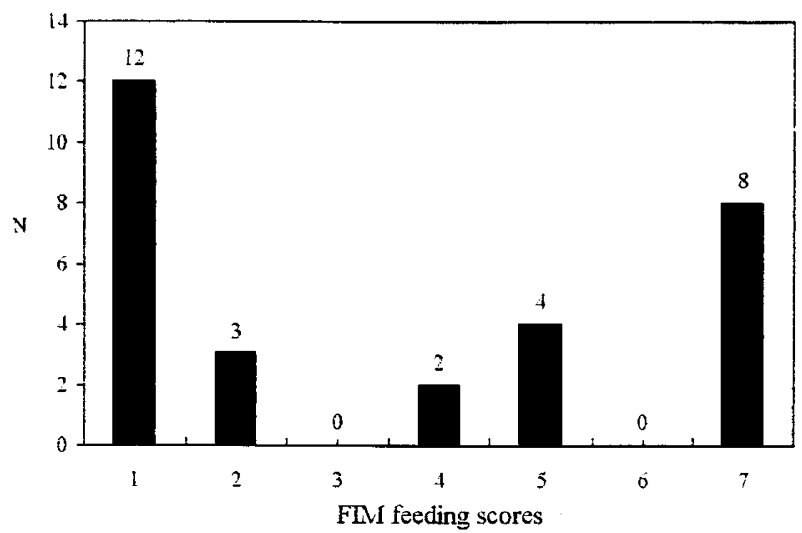

Figure 2 Distribution of patients according to FIM feeding score

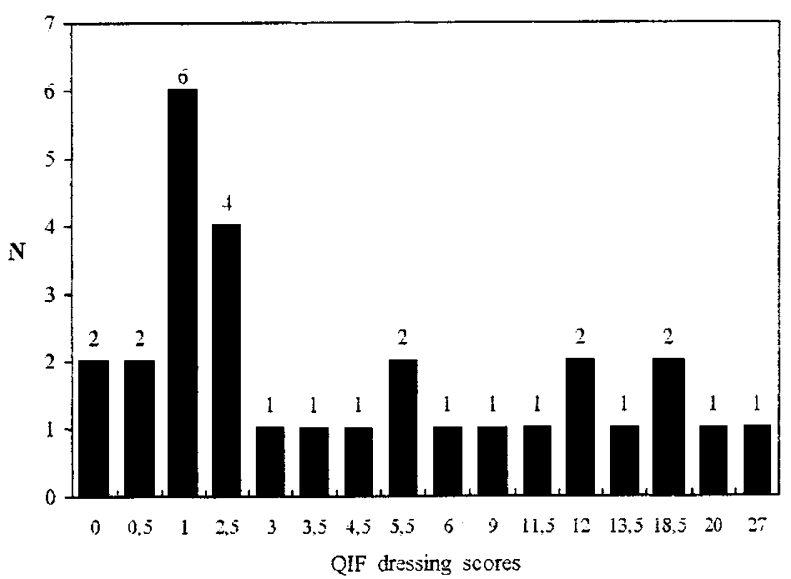

Figure 3 Distribution of patients according to QIF dressing score

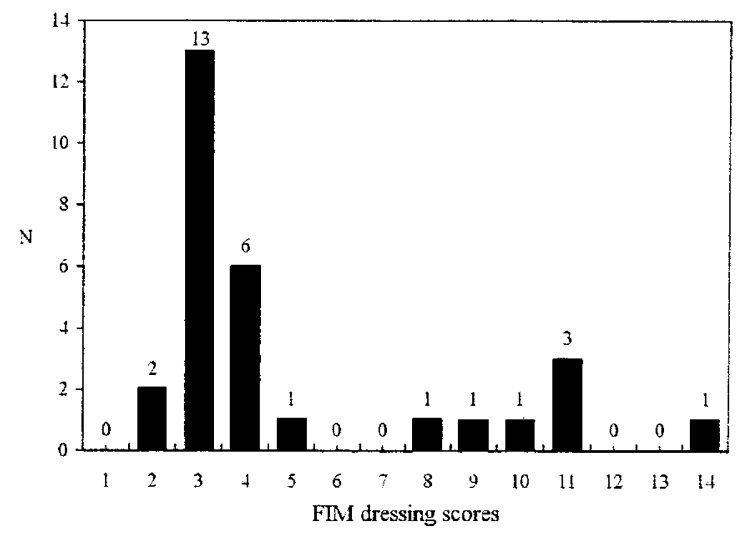

* The score includes the sum of the scores for dressing the upper(7) and lower

Figure 4 Distribution of patients according to FIM dressing score category questions eight and the dressing category pinpoints nine different items, each of these assessing a different aspect of function. This makes it possible, in these two QIF categories, to detect small differences among the patients and small gains made by each patient during rehabilitation process.

The QIF includes an extra category in its assessment of patient functional performance, that of bed activities. This is not part of the FIM assessment. The correlation of this category of QIF with ASIA motor score was 0.90 , which also accurately reflected the relationship between function and strength.

We assessed the patients first at admission and then again at discharge, and thus we were able to discuss the value of two tests in assessing outcome.

Both tests showed remarkable improvement in scores. The average gains in QIF and FIM scores was 30.12 out of 180 points $(16.7 \%)$ and 14.20 out of 126 points $(10.9 \%)$, respectively. However, when we correlated per cent recovery in motor scores to per cent gains made by each patient on total QIF and total FIM scores, we found a correlation with improvement in QIF, but not FIM, scores.

Gresham et al evaluated 30 quadriplegic patients using the Barthel index, the Kenny self-care evaluation test, and the QIF. ${ }^{1}$ They found the average per cent of recovery to be $36 \%$ using the Barthel index, 20\% using the Kenny self-care evaluation system, and $46 \%$ using the QIF. They suggested that QIF was the most sensitive functional test of those they assessed. In the present study, we compared QIF, a very detailed test, to FIM, a system used and accepted worldwide. The National Acute Spinal Cord Injury Study Group (NASCIS) considers FIM the standard functional test for assessing outcome. ${ }^{15}$ The American Spinal Injury Association also recommends the use of FIM for international standardization. ${ }^{7}$

Both the FIM and QIF tests were successful in terms of revealing outcome for complete and incomplete tetraplegic patients (Table 3).

In our series, both functional tests failed to indicate any significance in differences in age or length of hospital stay. Recovery in motor scores was not found to be significantly correlated to these differences either, however. On the other hand, time from onset of tetraplegia was found to be a significant factor in motor recovery, and this was reflected as functional gain only on the QIF test. We found QIF to be a much better indicator of motor recovery compared to FIM. Both tests are less dependent on age and hospitalization period, unless perhaps associated diseases are involved, which was not the case in our patients.

In conclusion, we can state that both the FIM and QIF are useful in assessing the complete or incomplete quadriplegic patient during rehabilitation. However, QIF is the more sensitive evaluation, since it can reflect small gains in function which parallel the small steps in recovery of strength. In terms of scores 
themselves, the numerical difference in recovery may not seem striking, ie. an ASIA motor score of 10 improving to 12 , but this actually represents a large (20\% in the example given) improvement. This degree of recovery is more sensitively reflected by the QIF than the FIM.

FIM is a very practical test, and inter-rater reliability is known to be high. ${ }^{14,15}$ In light of the findings of our study and the previous one by Marino et $a l^{4}$ certain additions to the FIM may be useful. In the feeding and dressing categories, one more specific item could be added and, much more importantly, the bed activity category could be included in the FIM as well. Further research is also essential.

We believe that with such contributions, FIM would be a more sensitive assessment, adding to its benefits as a very practical and reliable test.

\section{References}

1 Gresham GE et al. The quadriplegia index of function (QIF): Sensitivity and reliability demonstrated in a study of thirty quadriplegic patients. Paraplegia 1986; 24: $38-44$.

2 Granger CV et al. Outcome of comprehensive medical rehabilitation: Measurement by PULSES profile and the Barthel index. Arch Phys Med Rehabil 1979; 60: 145-154.

3 Roth E et al. Functional assessment in spinal cord injury: A comparison of the modified Barthel index and the 'adapted' functional independence measure. Clin Rehabil 1990; 4: 277-285.
4 Marino RJ et al. Assessing self care status in quadriplegia: Comparison of the quadriplegia index of function (QIF) and the functional independence measure (FIM). Paraplegia 1993; 31: $225-233$.

5 Ditunno et al. Motor recovery and functional prognosis in spinal cord injury. Rehabil Report 1989; 5: 1 -4.

6 Lazar et al. Prediction of functional outcome by motor capability after spinal cord injury. Arch Phys Med Rehabil 1989; 70: 819822.

7 American Spinal Injury Association. Standards of neurological and functional classification of spinal cord injury, revised 1992 ASIA, Chicago: $1-26$.

8 Hamilton BB et al. Interagreement of the seven-level functional independence measure (FIM). Arch Phys Med Rehabil 1991; 7: 790

9 Waters RL et al. Motor and sensory recovery following complete tetraplegia. Arch Phys Med Rehabil 1993; 74: 242-247.

10 Waters RL et al. Motor and sensory recovery following incomplete tetraplegia. Arch Phys Med Rehabil 1994; 75: 306311.

11 Ditunno JF et al. Motor recovery of the upper extremities in traumatic quadriplegia: a multicenter study. Arch Phys Med Rehabil 1992; 73: 431 - 436 .

12 Bednarczyk JH. Comparison of functional and medical assessment in the classification of person with spinal cord injury. $J$ Rehabil Res Dev 1993; 30: 405-411.

13 Allison GT. Initial factors predicting functional performance in patients with traumatic tetraplegia (letter). Paraplegia 1991; 29: 280.

14 Welch R et al. Functional independence in quadriplegia: Critical levels. Arch Phys Med Rehabil 1986; 67: 235-240.

15 Segal ME et al. Interinstutional agreement of individual functional independence measure (FIM) items measured at two sites on one example of SCI patients. Paraplegia 1995; 31: 622 631 